\title{
Téoros
}

Revue de recherche en tourisme

\section{Le tourisme dans la Caraïbe}

De la nécessité de sortir du tourisme de masse

\section{Olivier Dehoorne}

Volume 26, numéro 1, printemps 2007

Tourisme dans la Caraïbe : logiques régionales et perspectives de développement

URI : https://id.erudit.org/iderudit/1070988ar

DOI : https://doi.org/10.7202/1070988ar

Aller au sommaire du numéro

Éditeur(s)

Université du Québec à Montréal

ISSN

0712-8657 (imprimé)

1923-2705 (numérique)

Découvrir la revue

Citer ce document

Dehoorne, O. (2007). Le tourisme dans la Caraïbe : de la nécessité de sortir du tourisme de masse. Téoros, 26(1), 3-5. https://doi.org/10.7202/1070988ar d'utilisation que vous pouvez consulter en ligne.

https://apropos.erudit.org/fr/usagers/politique-dutilisation/ 


\section{Le tourisme dans la Caraïbe De la nécessité de sortir du tourisme de masse}

\section{Olivier Dehoorne}

\section{Le désir de Caraïbe}

L'imaginaire qui alimente la représentation touristique s'ancre dans l'histoire des explorations, des premières découvertes et de toute la littérature exotique et coloniale qui a marqué les derniers siècles. Ce sont les récits de la piraterie avec ces "hommes libres » qui écument la mer des Carailbes, ces artistes d'hier et d'aujourd'hui qui semblent vagabonder dans cet ailleurs. Le rêve et les désirs construisent un imaginaire de l'espace (Laplante, 1997) avec de lointaines îles accueillantes et généreuses, à l'image des femmes « indigènes » débordantes de sensualité.

Ce désir d'exotisme, quotidiennement entretenu dans les principales métropoles nord-américaines et européennes, s'affiche dans les stations de métro en rappelant à chacun l'existence de ce rêve à portée de la main. Les îles y sont réduites aux deux stéréotypes du sauvage et du sensuel par le biais des émissions dites de " téléréalité » comme celles qui consacrent le mythe de l'île de Robinson (du type Survivors), avec des candidats abandonnés sur des plages "désertes ", où, entre séduction, adultère et transgression (du type "île de la tentation "), les paysages tropicaux sont réduits à de simples décors artificialisés, mis en scène. Les nouveaux héros télévisuels gagnent leur éphémère notoriété dans ces "paradis tropicaux ", entre plongée dans les lagons et feux de camps sur les plages. La " pigmentation » locale est éventuellement introduite par quelques joueurs-acteurs, enfants d'émigrés, nés dans les métropoles occidentales.

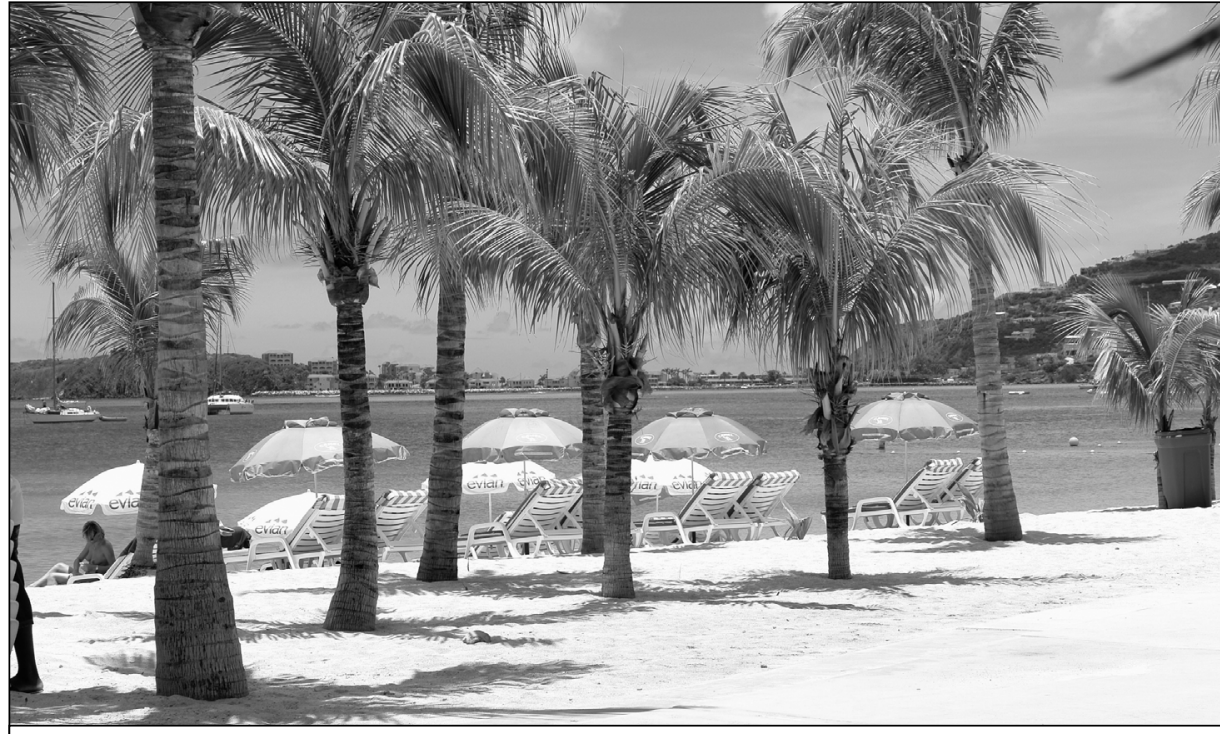

Plage de Punta Cana en République dominicaine.

Photo: www.residence-alizea.com

Les modalités de la communication évoluent, mais les fondements des discours sont les mêmes, qu'il s'agisse de la littérature populaire, des "zoos humains " (Bancel et al., 2002) ou de la téléréalité. La quête sensuelle des îles du plaisir s'impose. Les îles et les rivages de la Caraïbe, entre désir et fantasme, sont au cœur des convoitises.

Ainsi les terres caribéennes sont perçues à travers les habituels stéréotypes des plages de sable fin bordées de cocotiers, les aquariums naturels dans les lagons aux eaux cristallines associés à la luxuriance de la flore et à l'intensité des jeux de lumière. L'évocation de ces îles est indissociable du rhum, des carnavals, des musiques «tropicales». Au terme de la journée, les couleurs chaudes du soleil couchant se fondent dans l'ambiance festive des nuits caribéennes bercées au rythme du reggae, du calypso, de la soca, de la salsa et du merengue. Chacun a alors le sentiment de "connaître" ces îles où seules les sociétés locales éprouvent bien des difficultés à se reconnaître. "The Caribbean has been repeatedly imagined and narrated as a tropical paradise [...] a perpetual Garden of Eden in which visitors can indulge all their desires and find a haven for relaxation, rejuvenation, and sensuous abandon » (Sheller, 2003: 13). L'ignorance des réalités locales, la méconnaissance des données sociales et historiques sont à l'origine d'un malentendu touristique. Ces pays sont nés dans la violence : du massacre de tribus amérindiennes à la déportation d'esclaves africains, sans oublier l'importation de travailleurs indiens sous contrat. Les réalités caribéennes sont complexes dans cet espace marqué par une histoire coloniale inachevée avec des territoires aux statuts variables entre "possessions", "dépendances " et territoires indépendants "sous contrôle». 


\section{Caraïbe, Caraïbes et Grande Caraïbe : la construction d'un cadre régional}

Le terme d'espace "caraïbe » fut introduit par les États-Unis au début du XIX siècle à mesure de leur progression économique et militaire au-delà de leur frontière maritime méridionale (Gaztambide-Geigel, 1996 ; Knight, 2006). Sa définition a évolué au fil du siècle; elle fut d'abord entendue comme les territoires des Indes occidentales britanniques. Le concept était soutenu par les travaux d'intellectuels de la Caraïbe, comme E. Williams, W.A. Roberts ou encore G. Arciniegas qui posèrent les premières bases d'une conscience pan-caribéenne, anticoIonialiste et anti-impérialiste, dès les années 1940 (Girvan, 2001).

Dans les années 1970, deux approches s'opposaient: la Carailbe anglophone composée des îles anglophones, francophones, hispanophones et néerlandophones, plus les trois Guyanes et le territoire continental du Belize (Williams, 1970) et la Carailbe hispanique qui s'étendait du canal du Yucatan au golfe de Paria (au large du Venezuela), incluant les États riverains de la Carailbe, du centre et du sud de l'Amérique, en excluant El Salvador (côte Pacifique), les Bahamas (trop étatsuniennes) et les Guyanes (côtes Atlantiques) (Bosch, 1983 : 34). La vision élargie de la Caraibe qui se dessina au cours des années 1990 permit de concilier des enjeux économiques et culturels dans le contexte d'un nouveau projet politique par l'intermédiaire de l'Association des États de la Caraibe (créée en 1994) regroupant tous les États indépendants et les territoires non indépendants de l'ensemble du bassin de la Caraïbe. Les conceptions hispaniques et anglophones ont alors fusionné pour donner naissance à la " Grande Caraïbe " (El Gran Caribe en espagnol, The Greater Caribbean en anglais). Dès lors on utilise les termes de "Grande Carailbe " ou de "Carailbe " (au singulier) pour faire référence à l'ensemble du bassin. Toutes les îles de la Caraïbe (Bahamas comprises), le Mexique et l'Amérique centrale, le Panama, la Colombie, le Venezuela, la Guyana, le Suriname et la Guyane française sont inclus dans cet espace qui commence au sud des côtes étatsuniennes. La Grande Caraibe est une construction politique d'un espace en quête de cohésion.

\section{Les îles et les rivages de la Caraïbe : la délimitation de notre champ d'étude}

Sur le plan des études touristiques, par Carailbe nous entendons l'ensemble des îles et des rivages baignés par la mer Caraïbe en excluant les côtes étatsuniennes et celles des Guyanes (dont les modalités de développement relèvent davantage des problématiques amazoniennes). La zone considérée, peuplée de plus de 300 millions de personnes, couvre près de 4 millions de kilomètres carrés qui se composent d'une mosaïque de territoires aux statuts divers et aux niveaux de vie des plus contrastés (une dizaine d'États continentaux, 13 États insulaires indépendants et 16 territoires sous contrôle nord-américain ou européen). Les écarts des PIB (produits intérieurs bruts) par habitant sont de 1 à 137 (moins de 354 USD pour Haiti en 2001 contre plus de 48742 USD aux îles Caïmans). Les économies les plus prospères, telles celles des îles Vierges américaines et britanniques, des Bahamas et des Antilles françaises, côtoient des États indépendants plus pauvres.

\section{Carte 1}

\section{L'espace caribéen}

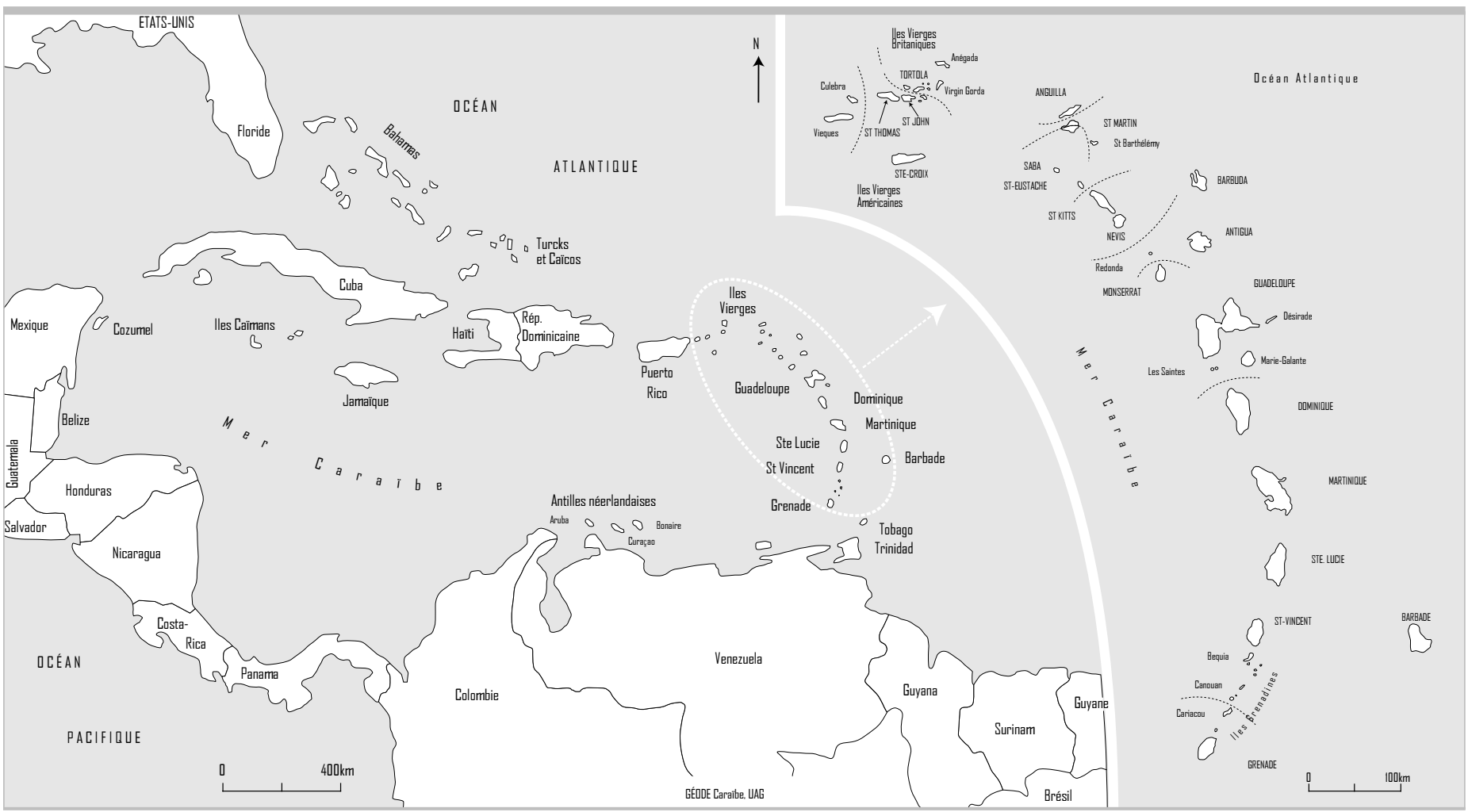

Source : Carribean Tourism Organization. 


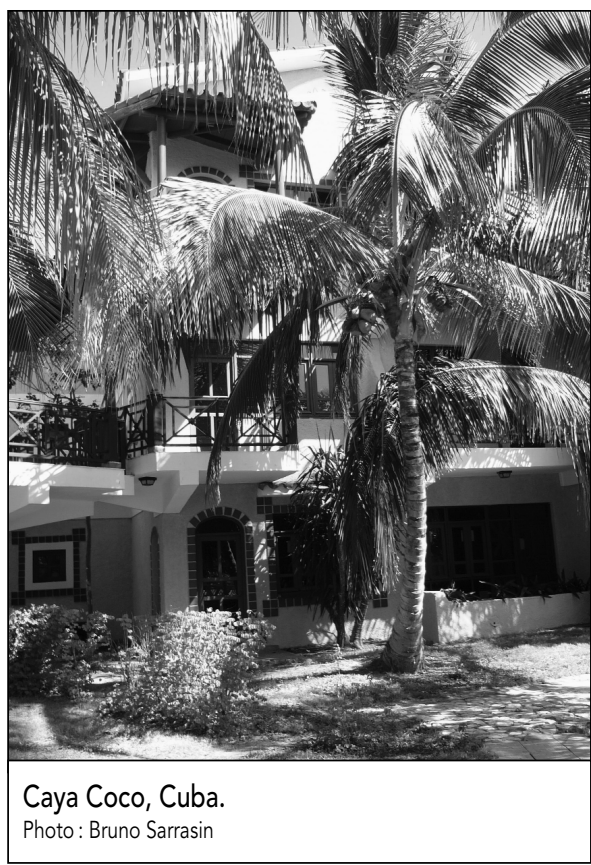

Ces terres bordées par la mer des Caraïbes constituent un champ d'investigation particulièrement riche. Ces territoires de l'Amérique tropicale des plantations partagent quelques caractéristiques communes essentielles: des économies dépendantes confrontées aux systèmes des plantations et des corporations multinationales, des sociétés marquées par l'esclave, la créolisation et la résistance populaire. Le tourisme est essentiel pour l'économique de la région. Leurs principaux atouts reposent sur une même représentation de la «tropicalité » réduite aux «3S» (Sea, Sun, Sand), avec un quatrième " $S$ " (le sexe en plus) pour les destinations motrices (Cuba, la République dominicaine et la Jamaïque en particulier). Les inégalités de richesses de ces sociétés contribuent à attiser les rivalités: tandis que les îles plus riches vantent leurs atouts sécuritaires (sécurité physique, alimentaire et sanitaire en général), elles doivent intégrer des coûts salariaux plus importants. À l'inverse, les îles en développement vendent des séjours peu chers facilités par de faibles charges salariales, souvent accompagnées de législations environnementales moins contraignantes. Et loin des discours incantatoires sur la coopération régionale et le tourisme durable par exemple, les rivalités économiques sont particulièrement vives dans cet espace où chacun entend s'imposer auprès de ses marchés émetteurs privilégiés, grâce à la commercialisation d'une offre uniformisée.

\section{Les problématiques autour du développement du tourisme dans l'espace caribéen}

Le premier texte du dossier (O. Dehoorne) propose un cadrage général sur les logiques spatiales du tourisme et le redéploiement des flux au sein de l'espace caribéen. D. Hiernaux, quant à lui, illustre les logiques du tourisme de masse à travers l'expérience mexicaine, en insistant sur les trois étapes du développement touristique dans la Caraïbe, la première étant celle du tourisme spontané qui investit les lieux au gré des opportunités, tout en posant les bases d'un embryon d'organisation à la fin de la première partie du milieu de $X X^{e}$ siècle. La deuxième est celle des planifications et des grands aménagements orchestrés par des gouvernements soucieux de faire du tourisme un secteur économique essentiel pour leur développement tout au long des années 1970-1980-1990, au gré des nouvelles indépendances (des petites îles anglophones) et de la reconversion des économies de plantation des grandes îles de la Caraïbe. La dernière étape, qui s'engage à partir des années 1990, est celle de la libéralisation des économies et de l'introduction d'investissements étrangers significatifs qui précipitent la course à la privatisation des lieux et des ressources. Ce sont les mécanismes de cette dernière étape qu'analyse M. Sheller avec l'étude de cas consacrée à l'île Vieques (Porto Rico) où la fermeture de la base de la US Navy est l'occasion d'une appropriation de cette île côtière à des fins touristiques. Dans cette Caraïbe confrontée à la finitude de son espace, chaque île et îlot est source d'intérêt: la mise en tourisme donne lieu à une théâtralisation de l'espace (qui hérite d'une nouvelle virginité) et à de subtils processus de fermeture des lieux en dépit des résistances locales (comme l'île Vieques).

L'approche de B. Sarrasin apporte un autre éclairage sur ce tourisme de masse considéré à partir des pratiques touristiques des Canadiens qui privilégient les principales destinations de la région (Cancun, République dominicaine et Cuba). Les logiques identifiées sont à l'image de celles des Nord-Américains en général ; les seules nuances concernent les volumes de touristes émis et quelques choix de destinations, les Étatsuniens privilégiant plutôt Porto Rico, la Jamaïque et les Bahamas que Cuba.
Les textes suivants, de K. Logossah et de J. Baldwin, mettent l'accent sur deux limites fondamentales des modèles de développement qui prévalent. Par l'analyse des logiques du tourisme de croisière, K. Logossah s'interroge sur les impacts économiques et les retombées effectives (en termes de revenus et d'emplois) pour les sociétés d'accueil. Les enclaves flottantes que constituent les paquebots de croisière sont devenues " des destinations touristiques en soi " que l'auteur qualifie de «territoires touristiques sans terre », mais avec leur lot d'incidences environnementales. La ressource touristique repose sur des richesses biophysiques spécifiques; les images touristiques leur accordent une valeur immatérielle supplémentaire, mais l'activité se développe en étroite relation avec ces milieux vulnérables, comme l'illustre J. Baldwin, à partir de l'étude de cas consacrée aux plages d'Antigua. Au terme de ce dossier, $\mathrm{J}-\mathrm{M}$. Breton ouvre une réflexion sur les perspectives de formes alternatives de tourisme, davantage en harmonie avec une approche durable du développement régional pour les États de la Caraïbe.

Olivier Dehoorne est maître de conférences à l'Université des Antilles et de la Guyane (Martinique).

\section{Bibliographie}

Bancel, N., P. Blanchard, G. Boetsch, E. Derro, et S. Lemaire (2002), Zoos Humains. De la Vénus hottentote aux reality shows, Paris, La Découverte.

Bosch, J. (1983) [1970], De Cristóbal Colon a Fidel Castro: El Caribe, Frontera Imperial, La Habana, Editorial de Ciencias Sociales.

Gaztambide-Geigel, A. (1996), « La invención del Caribe en el Siglo XX. Las definiciones del Caribe como problema histórico y metodológico ", Revista Mexicana del Caribe, vol. 1, n 1, p. 75-96.

Girvan, N. (2001), "Reinterpreting the Caribbean », dans F. Lindahl et B. Meeks (dir.), New Caribbean Thought, University of West Indies Press, St. Augustine WI Press.

Knight, F. (dir.) (2006), Beyond Fragmentation: Perspectives on Caribbean History, Markus Wiener Pub.

Laplante, M. (1997), L'expérience touristique contemporaine. Fondements sociaux et culturels, Sainte-Foy, Presses de l'Université du Québec.

Sheller, M. (2003), Consuming the Caribbean: From Arawaks to Zombies, Routledge.

Williams, E. (1970), From Columbus to Castro: The History of the Caribbean, 1492-1969, London, Harper and Row. 